Accepted on Synthese - December, 2018

DOI: 10.1007/s11229-018-02044-1

\title{
Affordances, context and sociality
}

\author{
Anna M. Borghi1,2 \\ 3 Department of Dynamic and Clinical Psychology, Sapienza University of Rome, Italy \\ 4 Institute of Cognitive Sciences and Technologies, Italian National Research Council, Rome, Italy.
}

Address for correspondence

Anna M. Borghi

Department of Dynamic and Clinical Psychology, via degli Apuli 1, 00145 Rome

Email: anna.borghi@uniroma1.it

\section{Acknowledgments}

I would like to thank Luca Tummolini for thoughtful comments on a previous version, and Laura Barca, Chiara Fini, Francois Foerster, Luisa Lugli, Giovanni Pezzulo, Laura Rio and Matt Sims, for discussions on affordances.

\begin{abstract}
Affordances, i.e. the opportunity of actions offered by the environment, are one of the central research topics for the theoretical perspectives that view cognition as emerging from the interaction between the environment and the body. Being at the bridge between perception and action, affordances help to question a dichotomous view of perception and action. While Gibson's view of affordances is mainly externalist, many contemporary approaches define affordances (and micro-affordances) as the product of long-term visuomotor associations in the brain. These studies have emphasized the fact that affordances are activated automatically, independently from the context and the previous intention to act: for example, affordances related to objects' size would emerge even if the task does not require focusing on size. This emphasis on the automaticity of affordances has led to overlook their flexibility and contextual-dependency. In this contribution I will outline and discuss recent perspectives and evidence that reveal the flexibility and contextdependency of affordances, clarifying how they are modulated by the physical, cultural and social context. I will focus specifically on social affordances, i.e. on how perception of affordances might be influenced by the presence of multiple actors having different goals.
\end{abstract}


Index

1. Introduction

1.1.Affordances: an embodied and grounded perspective

1.2. Affordances: automaticity and/or influence of task and context?

1.3. Aim of this work

2. Affordances and the physical context

2.1.One object, more affordances

2.2.Two objects, more affordances

2.3.Affordances in everyday contexts

2.4.Social invitations to respond to affordances in everyday contexts

2.5. Summary

3. Affordances and the social context

3.1.One object in the near space of others

3.2.Observing someone reaching an object

3.3.Grasping an object and speaking of object manipulation

3.4. Being asked for an object while reaching it

3.5.Placing vs. giving an object

3.6.Giving something to someone (feeding somebody) when we are asked

3.7.Giving and taking objects from gentle/rude others

3.8. Summary

4. Affordances, social context and joint action with one or more objects

4.1.Acting synchronously with the same object

4.2.Signaling during joint action with one or two objects

4.3.Acting synchronously and sequentially on two objects

4.4.Two objects and two people: perspective taking

4.5.Two objects and two people: social signals

4.6. Summary

5. Affordances, social context and social norms

5.1.Positive objects for ourselves

5.2.Ownership and affordances

5.3. Summary

6. Affordances and culture(s)

7. Conclusion 
Affordances, context and sociality

\section{Introduction}

\subsection{Affordances: an embodied and grounded perspective}

The environment in which we live is full of action possibilities provided by objects - the affordances (Gibson, 1979). Being able to respond adequately to object affordances is pivotal for our life. This paper will focus on how we represent affordances of objects, and especially of manipulable objects, and aims at highlighting their flexibility and contextual dependence.

The notion of affordance, originally introduced by Gibson (1979), in the last years has widely gained in popularity and it is largely debated in disciplines as diverse as psychology, cognitive science and neuroscience, philosophy and robotics. I will now outline the notion of affordance I will adopt, briefly referring to the theoretical debates in the field.

In the philosophical debate, two main positions exist: according to the first, affordances are properties of the environment relative to organisms. In Reed's (1996) account, affordances are resources that orient natural selection, since the environmental affordances are more persistent in space and last longer than the movements of organisms in the environment. "..the source of the psychological components of natural selection are the affordances of the environment. It is argued that these affordances select and shape animal behavior and awareness, not only on the time scale of natural selection, but also within more narrow time scales, such as that of ontogeny, learning, and individual behavioral acts" (Reed, 1996, p. 29). In other accounts, in which affordances do not orient natural selection, affordances are dispositional properties of the environment, that to be actualized need some animal properties. Such properties of animals can be abilities - e.g. the affordances offered by a mountain can be perceived by those who have the ability to climb (Turvey, 1992: Michael, 2000) - or body scale (Heft (1989), - e.g. the chair is a good sitting affordance for humans, owing to our body scale, but not for ants and worms.

According to the second position, proposed by Chemero (2003; 2009), "affordances are not properties of the environment; indeed, they are not even properties. Affordances.. are relations between particular aspects of animals and particular aspects of situations." (Chemero, 2003; p. 184). Perceiving affordances in his view corresponds to placing features, for example seeing that the current situation allows a certain activity, and it does not imply relying on previous knowledge: we perceive that it is raining now, and the current situation differs from all other situations. In his view, in cases like that a property - the fact that it is raining - is being ascribed to the environment, but not to a specific object. Affordances do not need to inhere in objects "Action is preceded by perception of a particular sort of environmental property, one that need not inhere in an objectan affordance." (Chemero, 2001,p. 112)

Even if the following section will mostly focus on the neuroscientific debate, I agree with Chemero's view that affordances are relations and that the whole system animal-environment should be considered (see also Stoffregen, 2003); at the same time, while I understand his emphasis on the current situation, i.e. on online processes, I will review evidence on affordances showing cases in which affordances inhere specific objects, and I contend that it is very important to investigate how semantic knowledge influences perception of affordances (see, for a recent debate on this, Osiurak and Badets, 2016; Buxbaum, 2017). 
In the very recent literature, a broader notion of affordances has been proposed (Bruineberg et al., 2018). This notion relates affordances to all forms of life, and to all the regularities and sociocultural practices that support our interactions with the environment (e.g. Rietveld and Kiverstein 2014); this view is contrasted with a narrower view (e.g. Golonka,2015), that intends affordances as action possibilities specified by the energy information present in the environment. I am sympathetic with the extension of the notion of affordances. The main part of this article will however focus on the narrower notion of affordances, i.e. on action possibilities (especially manipulation) afforded by objects in the environment. In the last part of the paper, however, I will consider also affordances in the context of social norms and cultural practices (see section 5 and $6)$.

Thus, the notion of affordance adopted here is broader than Gibson's one: first because it is less externalist, owing to the interest for the neural underpinnings of affordances (Bruineberg et al., 2018), second because it relates not only to motor actions but also to sociocultural practices linked to affordances. The notion of affordance used here is extended in a further, more subtle sense. Zipoli-Caiani (2014) argued that affordances emerge not only when action-related dispositional properties are present in the environment, but also when sensorimotor patterns can be detected in perceptual stimuli, as images and depicted objects (on the relationship between action and images with respect to the visual stream see also Freud et al., 2018). It has been clearly shown that pragmatic and semantic processing of action possibilities concur in determining pictorial experience: we perceive depicted objects as pictorial, not real objects; at the same time, however, they offer us action possibilities (Ferretti, 2018). It is thus possible to ascribe action properties to images, even if depicted objects cannot be peripersonally localized in the same way as real objects (Ferretti, 2016a). I think that it is crucial to assume that in many occasions we store information on how to reach and grasp objects, and that current perception of action possibilities is influenced and assisted by our previous experiences and stored knowledge. In the present paper, I will highlight the influence of long-term knowledge on current affordances and tool use. Storing such information involves also the recruitment of the dorso-ventral system, while the dorso-dorsal system (Rizzolatti \& Matelli, 2003) adjusts actions to the online perceived object, to its distance and size etc. (for detailed arguments on this and on the conjunct operation and integration of the ventral and dorsal systems, and of the dorso-dorsal and ventro-dorsal systems see Buxbaum, 2017; Buxbaum and Kalenine, 2010; Binkofski \& Buxbaum, 2013; Borghi \& Riggio, 2015; Ferretti, 2016b, 2016c, 2017; Orban \& Caruana, 2014; Sakreida et al., 2016; Zipoli Caiani \& Ferretti, 2017; for a recent special issue entirely dedicated to the topic see de Haan, Jackson, \& Schenk, 2018).

In the neuroscientific debate, the increased popularity of the notion of affordances is likely due to the spread of embodied and grounded theories, according to which cognition is grounded in perception and action system (Barsalou, 2008; Glenberg, 2014; Borghi \& Caruana, 2015). A particularity of these theories is that they intend the relationship between perception and action as a loop, in contrast with the previous view according to which it was seen as linear and sequential - perception came first, then the perceptual stimulus was processed, and then an action followed. The traditional view does not take into account that perception might vary depending on the aim of our actions and on the motor responses we are going to produce. The 
circular loop between perception and action is reflected in the notion of affordances - affordances are namely intrinsically relational, since they involve both perception and action, both the organism and the surrounding environment.

The study of affordances received further impulse by the discovery in the monkey brain of canonical neurons, that fire not only during grasping actions but also during observation of graspable objects, independently from the fact that the object will be effectively grasped (Sakata et al., 1995; Murata et al., 2000). Further results with humans suggest that a motor simulation is taking place: observing an object leads to activate the right way to grasp that object (Gallese \& Lakoff, 2005). Also due to the discovery of canonical neurons, recent neuroscientific literature on affordances both took inspiration from Gibson but also departed from him, underlying the importance of brain processes, investigating the neural basis of affordances (e.g. ; Grèzes et al., 2003) and dropping Gibson's externalist account (for reviews, see Thill et al., 2013; van Elk et al., 2014).

\subsection{Affordances: automaticity and/or influence of task and context?}

The history of psychological and neuroscientific studies of affordances of the last years can be divided into two big chapters or phases, one focusing on automaticity and the second on the influence of task and context on affordances activation.

Automaticity. Until 2004-2005 the majority of studies focused on automaticity of affordances. With automaticity I intend the fact that affordances are activated even if they are not relevant to the task at hand. From the late nineties, after some seminal studies by Ellis and Tucker, scholars produced evidence that objects affordances were automatically activated even when the response was not relevant for the context and the task. In a paradigmatic study, participants were required to perform a judgment on one object characteristic (e.g. decide whether an object was upright or reversed, or whether it was an artifact or a natural object), while affordance-based compatibility effect were found between other characteristics on the object (the object orientation, the size) and the motor response - for example, participants responded faster to a right handled object when pressing a right than a left key, or responded faster with a precision grip to small than to large objects (Tucker \& Ellis, 1998; 2001; 2004). The task did not require focusing on handle orientation, or on object size, but participants automatically did it, as revealed by the compatibility effect found. Consistently, it was stated that affordances were activated automatically, i.e. independently from the task. In this view, affordances (and micro-affordances) would be the product of long-term visuomotor associations in the brain (Ellis \& Tucker, 2001); this view has been philosophically defended showing that these associations may develop through motor experience of trial and error practice (Ferretti, 2017a).

Starting from the first decade of our century, studies started to show that affordances were variable, modulated by the task and by the context. Notice that the evidence showing that affordances activation is modulated by the task and by the context challenges the idea that affordances are automatically activated, but it does not necessarily contradicts it. It is indeed 
possible, that once we see an object all of its affordances are automatically activated, and then the context acts as a sort of filter, before selecting the response.

Another way to conceptualize this problem is following the distinction between "real" and "perceived" affordances introduced by Norman (1999). According to the author, perceived affordances are the most important ones for object design. For example, a graphical object on the screen would afford a clicking action; perceived affordances refer to the fact that that clicking on that location represents a meaningful, sensible action. Following Norman's terminology, it is possible that all real affordances are automatically activated, but that only affordances relevant to a given context have the status of perceived affordances, i.e. the actions they invite to perform are intended as useful and sensible.

Task. In the first study in which the automaticity of affordance activation was questioned Tipper et al. (2006) employed images of door handles evoking right- or left-hand reach-to-grasp actions. The authors showed that there was an affordance effect only when the task required to discriminate their shape and not their color. Furthermore, the effect was more marked when handles were in an active position, with which current action was implied. In the same vein, Pellicano et al. (2010) demonstrated with torches as stimuli that the affordance effect was modulated by the task and by the characteristics of the object: a motor simulation of handling the torch was activated only when the torch was switched on, and when the task required participants to discriminate object shape but not object color. Borghi et al. (2007) showed with a priming paradigm and a categorization task of pictures that the compatibility between the kind of grip (precision, power) and the object size was present only when the experiment was preceded by a motor training phase, in which participants observed images and reproduced precision and power hand grip. Overall, these studies show either that the affordances activation is modulated by the task and depends on its different depth level (it is present in a shape classification but not in a color classification task) or that specific motor programs are activated only when the motor system is previously activated, thus cannot be intended as fully automatic.

Context. More recently many more studies have appeared, that reveal that affordances are flexibly activated and modulated by the context (for reviews, see Borghi \& Riggio, 2015; van Elk et al., 2014). For example, grasping affordances of a spoon differ if it is presented together with a cup or with a sheet of paper (e.g. Borghi et al., 2012; Yoon et al., 2010); similarly, for objects characterized by multiple affordances the context can influence the kind of activated grip: for example, seeing a cork within a drawer evokes a power way of grasping (manipulation), while seeing it on a bottle activates a precision kind of grasping (function) (Kalenine et al., 2014).

Object characteristics. There are some cases in which the automatic activation of affordances might be impeded, or blocked, due to some object characteristics. One of these cases is represented by objects with broken affordances. For example, Buccino et al. (2009) presented right-handed participants with objects with broken handles, and stimulated with Transcranial Magnetic Stimulation (TMS) the hand motor area. They found that the Motor Evoked Potential area was larger when the handle was on the right, but only when the handle was not broken (Buccino et al., 2009). Another is represented by dangerous objects, for example objects with 
thorns: behavioral and EEG studies have revealed that we automatically tend to keep distance from dangerous objects, or to freeze in their presence, blocking our motor responses (Anelli et al., 2012; 2013a, 2013b; Liu et al., 2017; Liu, 2018). In cases like that of broken affordances and of dangerous affordances, two possible scenarios are open. In the first, only the affordances related to the observed object, for example the cup with a broken handle or the dangerous object, would be activated. In the second scenario, the object' standard affordances would be activated, then their activation would be inhibited and blocked because of new information (that the handle is broken or that the object is dangerous); information on the object and on its characteristics (broken handle, dangerousness) would be activated serially or, more plausibly, in parallel. Our data suggest that dangerous affordances are perceived immediately and quickly, and this renders less likely the second scenario (see Anelli et al., 2013a; Borghi \& Riggio for further discussion on this topic). However, the two cases can be quite different, because being able to quickly respond to/avoid dangerous objects might be adaptively more important than to adequately respond to broken objects. In order to conclusively determine which of the two scenarios occurs, further research is needed, that takes into account the time course of affordance activation and that takes advantage of interfering paradigms. If the second scenario occurs, then I propose that also in this cases objects affordances are automatically activated, but a competition takes place between conflicting information, leading to block our motor response.

How would competition between affordances take place?In the well-known model by Cisek (2007) the dorsal visual system specifies several possible actions within the fronto-parietal cortex, until more information is gathered through prefrontal areas and basal ganglia, that allows to bias the competition in order to select a single response. A competition between multiple action possibilities would rise, and the context would thus intervene to bias the competition and determine which affordance would win. The idea of competition is now well accepted, also thanks to the recent success of predictive coding views, that conceive the brain as a predictive machine (Pezzulo \& Cisek, 2016).

\subsection{Aim of this work}

In many cases our environment is crowded by objects, entities, people. The same object can evoke different affordances depending on the objects that surround it, the people who are near to it, their action intention, their willingness to perform an individual action or a joint one, the social norms that regulate the object's use, the cultural habit linked to its function. Strikingly, the majority of experimental studies so far have involved tasks that require participants to respond to the affordances of a single object. This is not necessarily wrong, but it can lead to misleading results. We namely perceive objects in contexts - objects are surrounded by other objects, and this has shown to modulate responses of participants. Furthermore, we often perceive objects in presence of other people, who have different intentions to act. The social dynamics evoked by objects might interact with their affordances, modulating the motor responses. But this is not the whole story. Objects use is regulated by social norms. Objects can belong to someone else. Evidence reveals that knowing that an object belongs to someone else automatically blocks activation of their affordances, even if the owner is not directly present on the scene. Finally, the 
environment is likely to be influenced by different cultures. In this paper I will review very recent literature showing that affordance activation is highly flexible and influenced by the context.

The aim of the paper is to propose that, when we observe single objects or more objects embedded in a scene or in a social situation, a competition between multiple action possibilities is activated. Such action possibilities would be evoked by the different affordances of the same objects, and by the relationship between objects characteristics and the physical, social and cultural context. The competition would determine a higher degree of uncertainty in more complex situations, and would be solved online, dynamically - for example, only affordances relevant to the current context will be selected. To substantiate my claim I will review recent evidence on affordance activation. I will briefly illustrate evidence on the influence of the physical context on affordances, while I will discuss more extensively how affordances are modulated by the social context. Clearly in a social context the degree of uncertainty between action possibilities can be higher, due to the difficulty in interpreting the intentions of others. In the case of the mere presence of another person, the affordances of the object activating our own actions would win. When the other person intervenes, the activated dynamics is more complex, and it is influenced by the aim of the action (giving, taking something from the other, performing independent actions on the objects or performing a joint action with him/her) and by the relationship between the two agents (collaborative, of friendship, or competitive). Notice that the idea of the competition among multiple affordances is not new (see Cisek, 2007; Pezzulo \& Cisek, 2016; Borghi and Riggio, 2015; Zipoli Caiani \& Ferretti, 2017).

However, it is important to understand when such a competition occurs, which mechanisms underline it, and how it is differently solved depending on the different situations and contexts.

2. Affordances and the physical context

\subsection{One object, more affordances}

Objects can have multiple affordances that might be compatible or conflicting. Conflict objects (Jax \& Buxbaum, 2010) activate competing action potentialities related either to manipulation or function; for example, a pen can activate a precision grip when we use it to write, or a power grip when we take it out from a drawer. Bub and Masson (2008) distinguish functional and volumetric gestures, the first associated to object use and the second used to pick up the objects.

Recent results show that the physical context can contribute to solve such a competition: the context acts on affordances selection, leading to activate either manipulative or functional grip on the object. Kalenine et al. (2014) presented participants with everyday scenes related either to manipulation/move or to function/use - for example, a kitchen timer could be presented in a kitchen drawer or on a kitchen counter, or keys could be displayed either in a desk drawer or inserted to desk lock. Participants had to perform a categorization task, deciding whether the objects were artifacts or natural objects, pressing a device with a precision or a power grip. Results showed a compatibility effect between the visual scene and the response; in particular precision 
grip was faster when objects were displayed in use rather than in move context. Further studies reveal how affordances are differently recruited depending on their distance from the body. In a first study by Costantini et al. (2010) participants were required to perform a grasping movement as soon as a go signal - a scene - appeared. The scene consisted of 3D objects (e.g. mugs) displayed either in the near, reachable space (30 centimeters) or in the far, non-reachable space (150 centimeters) of participants. The affordance effect, i.e. the compatibility between the response effector and the handle location (left/right), occurred only when the affordances were in the near space. A further study (Kalenine et al., 2016) demonstrated that reaching distance influenced perceptual judgments on objects. Such judgments were slower with conflict objects, i.e. objects that elicited both structural and functional affordances.

These results suggests that, while processing conflict objects, a competition between different affordances is activated. However, the context, i.e. the scene and the distance of objects from the body influence the competition: move-related scenes and use-related scenes elicited respectively power and precision grips and RTs of conflict objects were slower only when the objects were within reach.

Not only the physical, but also the linguistic context can modulate motor response to affordances. In two studies (Costantini et al., 2011a; Ambrosini et al., 2012) participants were shown a verb referring to function, manipulation, pointing or observation and had to decide whether it matched with the 3D object presented in the near or far space. The advantage of affordances in the near space was very pronounced with function verbs, still present with manipulation verbs and disappeared with pointing and observation verbs. Response were faster with action verbs when the objects were presented in the real near space, rather than in the perceived reaching space. These results indicate that the linguistic context influences the competition between object affordances: action verbs overall, but particularly verbs related to use, render responses to affordances faster than observation verbs, and promote the winning of near affordances over far ones.

The linguistic context can also influence activation of different kinds of affordances. Borghi and Riggio $(2009,2015)$ distinguished between two different kinds of affordances - more stable affordances, as those related to object size, that are worth to be kept in long-term memory, and more variable affordances, as those related to the current handle orientation of a brick, that might not be worth memorizing but are to be computed online. These two kinds of affordances might enter in competition; the winner of such a competition might be different depending on the context. While in online interaction with objects both variable and stable affordances are recruited, and the first likely prevail over the second, during language processing stable affordances are activated, i.e. affordances that refer to more stable characteristics of objects as shape and canonical orientation (Borghi \& Riggio, 2009).

\subsection{Two objects, more affordances}


We have seen that one object can evoke multiple affordances. The presence of two objects instead of a single one can both render the situation more complex, but also simplify it, providing cues for action. For example, seeing a spoon near a bowl might help us to understand that we might use the spoon to eat the soup. A number of studies have recently investigated the affordances elicited by pairs of objects, and how the competition between multiple affordances can be generated and solved in such situations.

In a variety of studies, Yoon, Humphreys and collaborators investigated the paired affordance effect (Yoon et al., 2010; Riddoch et al., 2011). They presented participants with pairs of objects, one of which was to be actively manipulated, while the other was passive: for example in the pair "spoon-bowl" the spoon is actively used, while the bowl represents the reference object. Correctly positioning objects for action and orienting it for use (e.g. a corkscrew going towards the top of a wine bottle) facilitated identification of active objects, even if briefly presented (Roberts \& Humphreys, 2011).

Xu et al. (2015) presented objects either in a correct location for action or in an incorrect one, they found a facilitation of the active over the passive object when positioned as if interacting (for example, with the spoon oriented toward the bowl). Importantly, the effect was found even if the objects were irrelevant to the task: participants were namely required to respond which shape, circle or triangle, was presented after the paired objects, aligned with each of them. The authors interpret the results in terms of a competition between action possibilities: all affordances from both objects would be extracted, then the active object would win the competition, while the response to the passive one would be interfered because it is incompatible with the response elicited by the object pair as a whole. Such an interpretation was confirmed by a recent Transcranial Magnetic Stimulation (TMS) study (Xu et al., 2017) highlighting the crucial role of the dorsal system in the paired affordance effect; in particular repetitive TMS applied on the left alPS reduced the interference effect in the responses aligned with the passive object.

Further effects of the presence of a second object on the affordances of the first were investigated by Borghi, Wheaton and Natraj. In a study by Borghi et al. (2012) participants were shown pictures of objects pairs and had to decide by pressing a different key whether the two objects were associated or no. The objects could have a functional relation (e.g. knife-butter), a spatial relation (e.g. knife-coffee mug) or no association. A hand could be presented, either simply near the object, or manipulating it with a manipulative/move grip or a functional/use grip. Results clearly showed an effect of context: in the use context manipulation hand postures were the slowest, while in the spatial context functional hand postures were interfered. Hence, in case of mismatch between the action suggested by the object and the context, response times were longer. Results can be clearly interpreted in terms of a competition between multiple action possibilities, in which those relevant to the current context win. Such a competition occurs only when the appropriate motor program had to be selected - they did not appear with foot responses. A following EEG study (Natraj et al., 2013) revealed that, while in the use context both functional and manipulative hand postures engaged early responses, in the spatial context data revealed extended neural response for the manipulative posture. This evidence is in keeping with the hypothesis that multiple affordances are competing, and that timing information is crucial to understand their interplay (see Young, 2006, for convergent evidence). A further eye-tracking study (Natraj et al., 2015) informs on how spatial and use affordances modulate visuospatial attention: results revealed that, in performing the task, participants focused more on the grasps in the use context 
and in the spatial context than in the incorrect context; the prolonged attention to the manipulative grasp suggests that they were struggling to interpret the action intention. Gaze focused primarily on the hand object (e.g. nail) and on the operant tool-end (e.g. hammer-head) more than on the graspable tool-end (hammer-handle): hence, the object seemed to prime attention when participants evaluated the relation between the two objects. Overall, these studies confirm that multiple affordances compete, and that both the physical and social context (the presence of other objects and the present of a hand with a different grip) contribute in selecting the response.

\subsection{Affordances in everyday contexts}

The studies illustrated so far show that context influences competition among affordances. However, the described studies typically employed pictures of more or less complex scenes to investigate contextual effects. A fresh way to look at affordances would imply investigating how we respond to them in everyday contexts - the playground, the kitchen, the workshop, the carpentry. I will make a couple of examples. In a first study, by Withagen and Caljouw (2017), the authors focus on playgrounds of Amsterdam designed by Van Eyck after the war. The authors show how the structures promote children's creativity, since they are not bounded to a specific and predetermined function, as slides and swings instead are. Limits of the affordances provided can be the regularity of the structures, in which blocks and bars are all equally distant from each other. Standardization is not only a limitation for children creativity but is also not considered desirable by children, even if their symmetry might appear desirable (Jongeneel et al., 2015). Creativity is thus linked to the possibility to let multiple affordances compete, without providing specific cues to solve the competition in a predetermined way.

Another example of a promising approach is outlined by a recent paper by Wokke et al. (2016). In this study the task is a standard cognitive psychology task, in which all the variables are controlled, but participants performed their task in a real environment, i.e. a kitchen vs. a workshop. Kitchen utensils and tools were displayed as go-stimuli; participants were required to respond to the object or to avoid responding when they saw an X. Results showed that response times were faster when the objects were congruent with the outside context (kitchen vs. workshop); a visual search control task ruled out the hypothesis that the effect was merely an attentional one. The same object, when congruent with the outside context, evoked a higher response conflict when participants had to withhold their response: ERP N2 component (active 200-300 ms after stimulus presentation) was more marked when participants had to refrain from responding when the target object was congruent with the context in comparison to an incongruent context. These results reveal that the real context influences the perception-action couplings, suggesting that all objects affordances were activated when the context was congruent, and highlights the high flexibility and contextual dependence of affordances activation. The approach outlined in this paper is a very promising one, since it combines a rigorous experimental procedure with the attention to the ecological character of the experimental setting.

As testified by the previously revised studies, in the last years the interest for the influence of the physical context on object affordances has increased. As the overview shows, a number of studies 
have investigated how displaying scenes where multiple objects, hence action possibilities, are present, affects affordances of the single objects. Whether the physical context influences affordances emergence or only their selection for response in a given context remains still an open issue, to be determined through further research: the context could namely act as an early or as a late filter: in the second case, only affordances relevant for the present context would be activated.

\subsection{Social invitations to respond to affordances in everyday contexts}

Separating the physical context from the social context can sometimes be misleading. Clark and Uzzell (2006), argue that the environment supports development through the interaction of physical and social affordances. Importantly, the social input of others can help us to fully benefit of the affordances offered by the environment. In a study on a Norwegian kindergarten Bjørgen (2016) showed that 3-5-years-olds need to be invited to play in order to fully exploit the affordances of the surrounding environment. The author compared two contexts, the outdoor space of the kindergarten, endowed with affordances given by outdoor toys (bucks, trucks), swings, climbing racks, and natural materials, as grass, sand, small hills, and natural environments of different kinds - for example, woods offering various playing opportunities, fields where to sky, run and go tobogganing. During free play the level of children's physical activity was not high, but it dramatically increased in the natural environment compared to the outdoor space of the kindergarten, where the environment had only fixed possibilities and was therefore likely perceived as more boring and predictable. More crucially, during free play the level of children's physical activity was not high, but when they were invited to play together with others they were able to exploit the potential affordances for physical activity present in the environment. Results of this study suggests that contexts perceived as novel and unusual offer more affordances, and also that affordances for physical actions are perceived more when others invite us to benefit of the stimuli offered by the external environment. When multiple affordances are activated, the competition is likely more difficult to solve for children and adolescents, who are more creative and less used than adults to stereotyped and fixed responses to objects.

\subsection{Summary}

In this section I have reviewed studies showing that affordances activation is influenced by the physical context: multiple affordances/action possibilities might be activated, but the setting, the distance from the body, the presence of further objects, even the linguistic context can bias the selection of the response and decide the winning affordance.

\section{Affordances and the social context}

Despite the growing interest for the relationship between affordances and context and despite the concurrent emergence of research in social cognition, an apparently trivial aspect has been neglected for years: the very fact that affordances might evoke different motor responses depending on the social context in which they are embedded. Only in the last years novel studies 
are emerging, focusing on this. I will review below recent studies on social affordances or, more generally, on how affordances are modulated by social situations and contexts.

\subsection{One object in the near space of others.}

An important contribution to investigate affordances as "windows to social cognition" (Costantini \& Sinigaglia, 2011) has been given by research focusing on how the activation of affordances is modulated by the distance of objects from the agent's body. Costantini et al. (2011b) demonstrated that the advantage in processing object affordances in the near space (see Costantini et al., 2010, 2011a, described above) occurred also when the object was outside the near space of participants, but was within the near space of an avatar. No effect was present when the object was outside the reaching space of participants but close to a virtual cylinder. Transcranial Magnetic Stimulation (TMS) results consistently showed higher MEPs when affordances were both in the reaching space of the individual or of an avatar (Cardellicchio et al., 2012). The authors interpret their results as suggesting the existence of a spatial mirror mechanism, motor in nature, allowing us to intend others as set of motor potentialities. Importantly, these potentialities are not necessarily accomplished leading to a real action, but can help us predict possible actions of the other. Such effects of the others are not limited to the peripersonal, near space: in a number of studies, Fini and collaborators demonstrated that the presence of another person with a potential to walk influences also the categorization of the space beyond reaching distance, leading to an extension of the space perceived as near (Fini et al., 2014; 2015). Furthermore, we judge the distance between another person and an object shorted than that between two objects, because we attribute to the other person the possibility to move (Fini et al. 2017). To the purpose of the present paper these studies establish a first important point: that we perceive affordances not only with respect to our own action possibilities but also to others action possibilities.

\subsection{Observing someone reaching an object.}

What happens when the object is not simply near to another person, but we observe someone reaching it? I will illustrate some studies, that investigate this. Jacquet et al. (2013) demonstrated that we are sensitive to the biomechanical constraints others have in responding to affordances. They asked participants to observe videos with an actor interacting with a tool. The tool had a handle that could be grasped either with a precision or a power grip, to pursue two different intentions: lifting the handle the box could be opened (o), while rotating the handle the light could be switched on (S). The complete action could be biomechanically optimal or suboptimal depending on the combination between the intention and the grip: for example, using the power grip to open the box was evaluated by independent raters as biomechanically optimal and did not imply any costs, as using the precision grip to switch on the light. In the videos shown, the probability to use optimal or suboptimal behaviors was manipulated: in the convergent bias condition $80 \%$ of the actions were optimal, in the divergent bias condition $80 \%$ of the observed actions were suboptimal, then a baseline condition was present. Videos representing the whole action were presented to induce a bias, then participants were shown videos of incomplete actions and had to predict the intention of the actor by pressing two different keys on the 
keyboard. Results revealed an important role of biomechanical factors: responses were facilitated in case of convergence between expectations derived from biomechanics and high probability of an optimal behavior, while responses were inhibited when a probabilistic bias was assigned to suboptimal behaviors. These results reveal that, when we observe another person interacting with an object, we concurrently activate the affordances of the object and we put ourselves in the other's shoes, simulating his/her hand grip on the object. Another study reveals that, when we observe someone grasping an object, we automatically adopt his/her perspective. Bloesch et al. (2012) found that, when participants observed another person reaching a distant object with a tool, they experienced a compression of the space and evaluated the distance closer that participants who observed an unsuccessful arm-reach. This suggests that the mere observation of the actions with an object leads us to automatically adopt the other's perspective (for a review on affordances and perspective, see Creem-Regehr et al., 2013).

When we observe someone reaching an object, the mirror neural system is activated, not only when we put ourselves in the other's shoes, but also when we activate a complementary action (Newman-Norlund et al., 2007). Ellis et al. (2013) showed participants with video-clips of a left- or right-handed reach toward a objects having a left or right handle. They asked participants to press a right/left key to determine whether the object they saw was a tool or a kitchen object. Responses were faster when the hand was opposite to that performing the reach, suggesting that participants activated a complementary action. Hence, the usual compatibility effect found with objects was modulated by the social context, even if the social context was completely irrelevant to the task. This reveals the profound influence of the social context on affordances processing. As Ellis et al. puts it, "The actual behaviour is the outcome of dynamic and sometimes conflicting influences from sources such as the affordance associated with objects, the goals of the agents, the spatial relations among the agents and objects, the actions of the agents, and so forth. The actual behaviour of an agent cannot of course itself be reduced to any of these parts in particular, and it will also tend to have reciprocal effects on those parts. Thus, to reach toward an object in a crowded material and social world is best understood as a dialectical process." (Ellis et al., 2013, p. 38)

In situations like those illustrated in these papers, the objects seem to activate affordances related both to ourselves and to the other. While in the evaluation task we tend to adopt the other's perspective, and we are influenced by biomechanical considerations regarding their grasps, in the task that involves a motor response a competition might take place: the objects evoke all possible affordances, both for us and for the other agent, but the presence of his/her hand constrains the action possibilities on the objects. Hence, of all possible actions, the action is selected, which is compatible with the object affordances and the presence of another person, i.e. the complementary action.

\subsection{Grasping an object and speaking of object manipulation.}

Consider now the simple situation in which we are going to reach an object on a table, and there is another person nearby. For example, we are in a crowded bar, and we are going to reach a cup of 
coffee the waiter has put on the counter. We can be friend of the person nearby or, or we might not know him/her. Either we or the other person are speaking and pronouncing the verbs "I/You grasp/take". In this case the two agents do not interact with each other using an object, even if each of them can speak. This was the setting of a kinematics experiment performed by Gianelli et al. (2013a). A first participant was required to reach and grasp an object in presence of a second participant who could be invisible (behind a curtain), sitting on his/her right/left side or sitting in front of him/her, either near or far from the table where the object was located. Manipulating the position of the second participant with respect to the object allowed the experimenters to independently control the influence of perspective (same or different from the agent) and of the reaching space (far or near from the object). While only the first participant was required to act with the object, each participant had to conjugate in the first or second person the verbs "to take" and "to grasp" (I take, you take, I grasp, you grasp). With this paradigm the authors intend to investigate the relationship between two kinds of social perspectives, the perspective induced by the object location with respect to the other and the perspective induced by the first and second person pronoun. Latency of maximal fingers aperture and velocity peak revealed shorter latencies in presence of a non-friend, suggesting that the other is perceived as potential competitor with respect to the object. This interpretation is supported by data showing that kinematics was not influenced by the position of the non-friend, but that the position of the friend played a role: latencies were shorter when friends were close to the object. The data confirm what found by Costantini et al. (2011): an object located in the near space of another person activates affordances. Importantly, however, in this study the two participants seem to enter in a potential conflict for the object: the agent interprets the other as potential competitor in grasping the object, particularly if he/she is not a friend and, if a friend, if he/she is near the object. To avoid that the other takes the object, the agent tends to act faster. Consistently, the agent acts faster when the other speaks and use the "I" pronoun: the sentences "I grasp" and "I take" seem to automatically suggest that the other is going to take the object, even if the agent knows that the second participant is simply conjugating a verb to accomplish the experimental instructions. Overall, this study suggests that, when we are going to grasp an object in front of us in presence of another person, we not only simulate the affordances for him/her, but we also plan our actions considering that he/she might enter in competition with us for its possession.

Hence, in presence of an object and another person who is speaking the affordances of the objects both for the agent and for the other are activated. However, since the agent intends to/is instructed to grasp the object, the competition is solved responding faster to our own affordances when we implicitly fear that the other will grasp the object, due to its position with respect to the object, or to the fact that he/she verbally signals that he/she will grasp it. Transposing this setting in a real life situation: In a bar, we drink faster from our cup when someone nearby says "I take", particularly if we do not know him/her.

\subsection{Being asked for an object while reaching it.}

When we are going to reach and grasp an object, communicative signals from others influence our interaction with it. Sartori, Becchio, Bulgheroni and Castiello (2009) found that an unexpected social request, consisting in a hand expressing the will to receive the object, influenced the task of placing the object on a platform, leading to a deviation of the trajectory toward the other's hand. The effect was not present in a control condition, in which another person was present but did not express any communicative intention, his/her gaze was not available, and the human hand was replaced by a robotic hand (see also Becchio, Sartori, Bulgheroni and Castiello, 2008a). In this case the agent's behavior was not only influenced by object's affordances, but the unexpected social 
request competed with the activation derived from affordances, leading to change the originally planned trajectory to reach for the object.

We can interpret this result arguing that, when the second agent does not express any request, no competition between the affordances of the object for the self and for the other takes place, likely also because the object is too far outside from the other's reaching space to generate affordances.

\subsection{Placing vs. giving an object}

Consider now what happens when we intend to interact with others by means of an object - for example, when we grasp an object in order to give it to someone else. Do we respond to object affordances in the same way if we give an object to another person or if we simply grasp it to move it somewhere else, and does the attitude of the other person influences our way of interacting with the object?

In the studies described so far participants simply had to grasp objects on their own, even if others were present and if they manifested the intention to grasp the object. Two views are in contrast: the view according to which the mere presence of others changes performance and the view according to which performance changes only when a physical interaction occurs between two agents in relation to an object (review in Becchio, Sartori, Castiello, 2010). To disentangle the effects of the mere presence of others and of the interaction on kinematics parameters, in a study by Becchio et al. (2008) three conditions were contrasted: an individual condition, a condition where a passive partner was sitting in front of the agent, and a social conditions. Participants were required either to grasp an object and to locate it on a concave base, or to give it to another person sitting near them. Kinematics of the reach-to-grasp phase revealed that in the social condition fingers were closed slower, suggesting a higher accuracy in dealing with the object while interacting with the other. As to the place phase, the longer deceleration phase revealed again a more careful attitude in the social condition. Overall, these studies reveal that, when the object has to be given to another person, it is reached, grasped and placed more carefully, in order to allow perfect tuning with the other.

\subsection{Giving something to someone (feeding somebody) when we are asked}

Further kinematics studies investigate a special way to give objects to others: they focused on the feeding behavior. Ferri, Campione, Dalla Volta, Gianelli and Gentilucci (2010) found that when participants reach, grasp and place a piece of food into the mouth of a human compared to the mouth of a non-human receiver the accuracy in movement execution increases (slowing down of the reaching and the placing components). Hence the same object is handled differently depending on the receiver. In a following study with the same paradigm, Ferri, Campione, Dalla Volta, Gianelli and Gentilucci (2011) confirmed that movement accuracy is higher in presence of a social request, i.e. the mouth aperture signaling the willing to be fed, even when the task does not involve feeding but reaching another part of the receiver's body. Such a social request was ineffective when the receiver was blindfolded, i.e. participants were precluded from observing his/her gaze. The authors propose that the gaze works as a cue suggesting that the social request is intentional and deliberate, thus making it effective. A similar effect of gaze during interaction of two people with two objects was found by Scorolli et al. (2014) (see description later). Overall these results reveal that perception of affordances varies depending on the social context. In presence of a social request coming from a human, and by concomitant gaze suggesting that the request is intentional, a social affordance is activated, that modifies the kinematics parameters, leading us to be more careful and accurate in interacting with the object to feed him/her. 


\subsection{Giving and taking objects from gentle/rude others}

When interacting with an object and with others, our motor response on the object varies depending on the attitude of the other toward us. Di Cesare et al. (2017) performed a kinematics study in which participants showed a video with a male and female actors interacting with an object. In a first condition (giving request) one actor asked for a bottle, either simply inviting the other with a gesture (visual modality) or asking it verbally ("give me", auditory modality), or performing both actions (mixed modality). The same was true for a taking request. The crucial manipulation pertained the style of the request, that could be either rude or kind. If the video displayed a giving request, participants were required to reach for, grasp, and move the bottle close to the monitor, while if it displayed a taking request, participants were required to reach, grasp, and move the bottle close to their body. Kinematics results indicate that, while responses were not modulated by the modality in which the request was presented (visual, auditory or mixed), they were influenced by the rude or gentle character of the request: rude requests elicited larger trajectories and higher velocity of the reach component, a larger maximal finger aperture of the grasp component, and earlier grip closure. The kinematics parameters reveal, in line with other studies, that a higher accuracy in the interaction is present, when the other is perceived as open toward the interaction. Overall, the studies involving giving and taking objects reveal that the responses to the object is influenced and modulated by the kind of social interaction: in case of a potentially positive social interaction, a higher movement accuracy is present.

\subsection{Summary}

In this section I have reviewed studies showing that affordances activation is influenced by the social context. First, we have seen that we perceive not only our own action possibilities but also other's action possibilities, for example when we see objects in the peripersonal space of others, or when we observe somebody reaching objects. Second, we have seen that, when we interact with somebody, for example to give him/her an object, social affordances arise, and we tend to be more accurate in interacting with the object the more the other is willing to interact with us, and gentle.

4. Affordances, social context and joint action with one or more objects

So far we have considered situations in which either one agent acted with an object in presence of another person, or he/she asked him/her to receive an object, or he/she gave him/her an object. The actions of receiving and giving clearly involve two agents, and their collaboration. We have seen that, when we have to give (or take) something from someone else, our actions are particularly accurate, especially if the other is a human who expresses an explicit request or a request that we can classify as intentional, and if he/she is our friend or is kind toward us. 
Consider now objects that require a collaborative action of two or more agents to be acted with, i.e. cases in which both agents have to interact with the same object at the same time. For example, two people have to lift a heavy table together: they have to synchronize their movements, performing complementary actions on the object. Different examples are multiple players who take turns in throwing a dice, or people who have to tide up a room together - in the first case they act on the same object in sequence, taking turns, in the second case they act on different objects following a common plan. I will now review some studies on joint actions performed with one or more objects. While the analysis of the mechanisms underlying joint action is beyond the scope of the current article, I will focus on how the motor interaction with an object is modulated by the fact that two agents have to concurrently interact with it. We will see that, when two or more agents are going to act with one or more objects, the agents provide each other signals to facilitate prediction of their action and to render the interaction smooth; furthermore, the relationship between objects can provide the context helpful to infer the action intention of the other agent.

\subsection{Acting synchronously with the same object}

In a variety of studies Sacheli, Candidi, Aglioti and collaborators explored how participants synchronize their movements with those of an avatar, manipulating a bottle-shaped object in a complementary fashion. In a recent study with limb apraxia (LA) patients Candidi et al. (2017) demonstrated that social affordances can facilitate the motor responses of patients. Participants were submitted to two different conditions: in the Interactive condition they were required to synchronize their movements with those of a virtual partner performing the same or a different movement, without knowing in advance which movement to perform; in the Instructed condition they were told whether to perform a power or precision grip, independently from the action of the other. Limb apraxia patients were more asynchronous than controls in the instructed condition, while the performance of the two groups did not differ in the interactive condition. The activation of social affordances, and the necessity to adapt online to the action of the other, rather than to follow abstract movement instructions, rendered LA patients more competent. The authors interpret their improvement in terms of the 'affordance competition hypothesis' (Cisek, 2007); possible competing actions are activated by the object and by presence of the other. This case of joint action clearly differs from the previously described situations in which the presence of another person while reaching for objects activated a competition leading to a quicker and less accurate grasping action (e.g. Gianelli et al., 2013a). Likely the presence of the other in this case, rather than activating competition, increased the salience of the objects affordances, rendering the interaction with the object smoother and improving competence in grasping.

Similarly to what happens when performing interactive actions like giving and taking, the interaction with the bottle differed depending on the relationship between agents. Sacheli et al. (2012) manipulated the kind of relationship between participants who had to synchronously reach and grasp an object with the shape of a bottle. After having performed personality tests, half of the participants were told that the other did not esteem their personality and interests. Then they had to perform the joint action on the bottle; depending on the sound they were given, they were 
told either to free interact with the bottle, or to grasp either the upper or the lower part of it (guided interaction). Participants with a negative relationship improved their performance in guided interaction at the expenses of the free interaction; coherently with this, kinematics analyses revealed that they adapted less to the behavior of the other participant, as revealed by the absence of movement corrections and by the low motor variability, suggesting that they performed the task in a more individual way.

Overall, these studies show that social affordances emerge during synchronous interaction of two participants with the same object. The presence of social affordances facilitates movements of apraxic patients with the object and is modulated by the interaction between the partners - the adaptation and synchronicity in movements between participants is maximal when the relationship between them is positive. Hence, the pattern of movements signals the willingness to cooperate or not while interacting with an object.

\subsection{Signaling during joint action with one or two objects}

When people have to coordinate themselves in order to act on the same object (e.g. performing a joint action on a bottle), or to perform a coordinated action with two objects (e.g. giving our cup to another person, who has to pour tea in it), they provide signals to the other through their actions/movements on the object. An example is a study by Sacheli et al. (2013) in which participants performed a joint-action task with a bottle-shaped object; during the task they were required to adopt either a leader or a follower role. In the first case they were instructed on where to grasp the object, while in the second case they were told to synchronize their movements with the other using imitative or complementary actions. When invested in the leader role, participants tended to use signaling strategies in order to render their movements more easily predictable: for example, kinematic peaks were reached more slowly, movement features were emphasized and movement variability was reduced.

Signaling strategies of this sort emerge also when participants are invited to give or take an object putting it near another person, without directly interacting with him/her. Scorolli et al. (2014) had an experimenter performing a functional or manipulative posture on everyday objects, as cups, and participants moving another object (e.g. tea-bag, tea-pot) toward the other or toward themselves. Results showed that participants used the hand posture of the experimenter grasping a cup as a signal to convey whether the experimenter's next action would be individual or directed to them (social), i.e. as signals provided to help the other to predict his/her own action.

Overall, these two studies indicate that in presence of objects with which two participants have to interact, they signal to each other which kind of action they are going to perform. They respond simultaneously both to the object and to the other. Importantly, different neural circuits are activated when responding to object affordances and when performing a joint action with an object. In order to verify this, Sacheli et al. (2015) asked participants to perform precision vs. power grips on a bottle-shaped object in synchrony with a virtual partner they saw on a screen, performing either complementary or imitative actions. Using cTBS (continuous Theta Burst 
Stimulation) they found that inhibition of left aIPS (anterior Intraparietal sulcus) reduced the ability to synchronize with the partner during complementary but not imitative actions.

\subsection{Acting synchronously and sequentially on two objects}

Consider a situation in which participants have to act on two objects either simultaneously or in sequence. Pezzulo et al. (2017) asked participants to lift glasses from each other trays either simultaneously or in sequence. Results showed that in case of simultaneous action, it was easier to keep the tray balanced. This reveals that performing the action with two objects at the same time is less costly and it implies benefits for both individuals involved in the interaction. The authors interpret the results arguing that the two actors do not have separate action plans, but a coordinated plan for bimanual actions in which the agents do not need to develop a separate plan for themselves and the other. This study is important since it shows that the presence of social affordances can lead to a simultaneous action, and that such a simultaneous action is less costly and more efficient than a sequential action.

\subsection{Two objects and two people: perspective taking}

We have seen that joint actions leads to activate social affordances. Consider now the situation in which what evokes affordances is simply the relationship between two objects in presence of a person. Tversky and Hard (2009) presented scenes in which two objects coexisted and asked participants to describe their spatial relation (e.g. "In relation to the bottle, where is the book?"). In some conditions, another person sat behind the objects, either looking or reaching for one of them. The produced relation was coded as conveying an egocentric (e.g. right), allocentric (e.g. left) or neutral perspective (e.g. next to). When in the scene another person was present, about $25 \%$ of participants adopted the perspective of the other, independently of whether the other was looking at the object or going to grasp it. The tendency to adopt the other's perspective was more marked when questions related to action were introduced (e.g. 'In relation to the bottle, where does he place the book?"). Overall, these results reveal that the simple presence of another person might reframe the way in which we think about relations between objects. Spontaneously taking the other's perspective might be functional to a successful interaction with the object and with the other, helping us to comprehend the other's action intentions.

\subsection{Two objects and two people: social signals}

The relationship between pairs of objects in presence of another person can suggest whether to respond to object affordances to perform a collaborative action or to simply observe the individual action of another person. To investigate the influence of relations between objects in a social context Scorolli et al. (2014) asked participants to grasp and move an object in front of them (e.g. a glass), either moving it toward themselves or away from their body, toward the experimenter. The experimenter, sitting in front of them, manipulated the second object (e.g. a can) using a functional vs. a manipulative grip/hand posture and looked directly at the participant or not. The pairs of objects could be unrelated (e.g. can-toothbrush) or related through a spatial relations 
(can-knife) or a functional one. Functional relations were distinguished into two kinds: they were distinguished in functional-individual (can-straw), related to an individual action, or functionalcooperative (e.g. can-glass), involving a possible social interaction. In the case of can-glass, we can use the can to pour its content in the glass of another person, while usually we use the straw to drink from our own can. When the participants were required to give the object (e.g. the can) to the experimenter, their wrist acceleration peak was reached faster when the experimenter manipulated the second object in a functional way, as if to use it for himself, and MFA was reached faster when the two objects had a functional individual relation, i.e. they did not evoke a collaborative action. In other words, participants inferred from the other's posture that the intention of the experimenter was to use objects on his own without involving them; more crucially, they inferred from the combination of the two objects that a cooperative action would not take place, hence they responded quicker, without preparing them to an accurate cooperative action. When participants had to move the object away from the experimenter (getting), when he directly looked at them MFA was greater when he adopted a manipulative than a functional hand posture, suggesting that he would let the other interact with the object rather than use it on his own. In other words, participants were more accurate in their relationship with the other, and used their actions on the object to signal their intentions. Well known signaling situations are the use of motherrese or motionese, i.e. the tendency of mothers to demonstrate objects use to infants with more enthusiasm, greater repetitiveness, slower movements, closer proximity and longer gazes to the infants (e.g. motherese, motionese: Brand et al., 2002; Brand \& Shallcross, 2008). In this case it is not one of the participants (e.g. the mother) who aims at increasing the social cohesion in the relation with the other, but the disposition of the objects themselves - and the hand posture of the experimenter - work as implicit and not deliberatively controlled signals favoring an individual over a social action.

\subsection{Summary}

In this section I have reviewed studies showing the effect on affordances activation of a special kind of social situation, that of joint action, in which participants acts synchronously or sequentially on one or more object. We have seen that participants signal to each other their action intentions, to render the interaction with objects smoother, and that signaling reflects the quality of the interaction between the participants.

\section{Affordances, social context and social norms}

When an object is positioned between two people, it affords actions to both of them; we have seen that each person will also activate the affordances of the object for the other, automatically infer the other's intention to act, and calculate his/her own possible actions with the object as a consequence. In many cases the relationship with objects is regulated by social norms. Objects can have a high value, and we might want to take possession of them. In some cases, objects might be owned by someone else (Tummolini et al., 2013). I propose that in these situations all affordances 
of the objects are activated and either potentiated, in case of "free" valuable objects, or blocked, in case of objects owned by others.

\subsection{Positive objects for ourselves}

Some studies reveal that humans tend to attract valuable objects for themselves. In a seminal study, Chen and Bargh (1999) have shown that participants tend to move a lever toward themselves when processing positive words, and away from themselves when responding to negative ones. The advantage of positive stimuli when associated to the self is present also when participants are required to take an objects for themselves, and giving a different object to others (Gianelli et al., 2011; 2013b). This suggests that, when a valuable object is present that does not clearly belong to anyone, a possible competition with others is activated.

\subsection{Ownership of objects}

Consider a situation that more subtly evokes a social context, and a norm respected in that context: imagine participants having only one cup in front of them. Depending on whether the cup belongs to them or to another person, the cup evokes different motor responses. Evidence has revealed that the sense of ownership is grounded on a basic mechanism, activated automatically even when the task does not refer to it (Scorolli et al., 2017). In two experiments Constable et al. (2011) offered participants a cup they could decorate, and then keep. When required to lift the experimenter's cup and their own cup, they were more careful in handling the experimenter's cup, moving it toward her, while they lift stronger their own cup, tending to move it toward their own body. Hence, the same cup, with the same affordances, elicited different motor responses depending on who was perceived as the cup's owner. A more in depth investigation of the kinematics effects revealed that the acceleration effect in lifting is directly linked to the presence of another person, while the effect on the trajectory was linked to object ownership but it did not necessarily imply the presence of another person(Constable et al., 2014). The effect of ownership was even more marked in a second experiment, in which the authors performed a standard compatibility effect task. Participants had to respond to the color of the cup's handle by pressing either a left or a right key on the keyboard. Response time analyses revealed a spatial compatibility effect with participant's cup: response times were faster when the location of the handle and that of the key to press (left vs. right) corresponded, indicating that observing the cup evokes its affordances. Importantly, such an effect was absent with the other's cup, suggesting that the motor response elicited by affordances was not activated for objects that were perceived as belonging to others. In a further study, Constable et al. (2016) asked participants to pass mugs to a friend or to an experimenter, then they measured the average difference in angle between the passed mug and the receiver's hand. When they passed their own mug participants tended to orient its handle less toward the others than when passing a mug owned by their friend or by the experimenter. This suggests that, while in joint action participants tend to facilitate others in dealing with objects, object ownership can modulate and influence such a behavior. Sevos et al. (2016) had participants perform a stimulus-response compatibility task. They were presented with a prime reporting either their surname or an imagery surname, used to evoke the sense of ownership on the object, followed by upright vs. reversed objects with a left- vs. right-oriented 
handle. RTs of participants were shorter in case of correspondence between the handle orientation and the key to press, but only when the prime was the participant's surname. When the prime was an imaginary surname, the effect of affordances disappeared, in agreement with Constable's results showing that affordances are not elicited when objects are owned by others.

Overall, these studies show that the activation of affordances is not present when objects are perceived as belonging to someone else, because they had been created and modified by someone else, because they were preceded by the name of their possible owner, or simply because participants were told that they did not belong to them. Hence, activation of affordances is not automatic but influenced by social norms. Such an intuition was present already in one of the first studies addressing affordances from an ecological perspective: "that pen on the desk may be graspable for me, given its diameter in relation to my grip, but because it is resting on the desk of the president of the college, it is not a pen I ought to pick up. From the standpoint of motor action, the pen does afford writing with, but from the standpoint of action in social context it does not." (Heft, 2003, p. 158).

It would however be important to understand the mechanisms underlying the described results, and to clarify whether these results are the product of a conflict. Further research should clarify, with precise timing analyses, whether objects affordances emerge and then are blocked due to the social norms (e.g., "do not take objects belonging to others") or whether the social norm acts as an early filter and completely impedes the emergence of affordances.

\subsection{Summary}

In this short section I have reviewed studies showing cases in which responses to affordances are ruled by social norms, as the case of object ownership.

\section{Affordances and culture(s)}

Affordances are often considered as a matter of the physical environment. But the history of affordances can be considered also as the history of our progressive adaptation to a social and cultural specific environment. Affordances are learned not only through physical interaction with objects, but also being exposed to observation of the conventional use of objects (Raymond et al., 2017). Affordances are often described as dynamical. The distinction between learned affordances and affordances derived from online interaction with objects has been addressed by many authors. For example, Norman (1999) distinguishes between physical affordances or "perceived affordances", mediated by the dorsal system, and "learned affordances", linked to conventional use of tools. He claims: "Don't confuse affordances with conventions. Affordances reflect the possible relationships among actors and objects: they are properties of the world. Conventions, conversely, are arbitrary, artificial, and learned. “(1999) Scholars seem to converge on the view that learned affordances are represented more ventrally in the brain. For example, Young (2006) proposes that damages to the semantic ventral system create problems with functional affordances. Patients with unpairment of the ventral system are unable to use tools in a conventional way, but still able to respond to tools, reaching and grasping them correctly. In a 
similar vein, Borghi and Riggio $(2009,2015)$ propose the distinction between stable and variable affordances. Stable affordances emerge from more stable knowledge on objects, like information on size, while variable affordances from more variable information, as that on the handle orientation. Consistently, a meta-analysis on $\mathrm{fMRI}$ studies reveals that the first are represented more ventrally than the second (ventro-dorsal vs. dorso-dorso system, Rizzolatti \& Matelli, 2003) (Sakreida et al., 2016). The distinction between manipulative and functional grips activated by affordances (see for example Jax and Buxbaum, 2010; Kalenine et al., 2014) also relates to the difference between affordances of novel objects and affordances of objects we have experience of. Once we first encounter objects, only manipulative affordances are activated. With time, we experience and learn their function, and functional learned affordances are activated too, competing with the first. The competition is often won by functional affordances, more frequently used in interacting with the object, but there is a contextual modulation, as shown in the first part of this paper.

Hence, many authors have focused on the distinction between affordances derived online, from the current interactions with objects, and affordances that reactivate previous experiences with objects. Strikingly, however, while the dynamics underlying online activation of affordances has been investigated, and some studies focus on the role of physical context in affordance activation and modulation, the issue of how affordances are influenced and modulated by the cultures and the habit of different social groups is neglected in current literature. We are not speaking about conventions, but about affordances, even if of learned affordances - we are speaking about the fact that in Western and Eastern societies different tools are used, and activated, to eat spaghetti, and that forks might activate eating affordances in Western societies more than in Eastern ones.

Let us start considering the very simple fact that the majority of environments with which we interact are modified by humans and differ depending on the culture in which they are immersed. In the recent years much evidence has revealed that the different spoken and signed languages influence the way in which the world is perceived, leading to a revival of Whorfian view (e.g. Casasanto, 2008). Similarly, the way we respond to affordances can be influenced by the characteristics of the physical environments we experience in our cultural milieu. Consider the well-known differences in attention pattern of Western and Eastern participants: the first tend to focus attention on objects, the second on the background elements. In an influential study Myiamoto et al. (2006) investigated whether these differences in attentional patterns are influenced by the environment in which people grow up. They sampled pictures of scenes of hotels and schools in cities of different size in Japan and United States, and asked participants to evaluate their complexity. Japanese scenes were evaluated as more complex and ambiguous than American ones, and East Asian participants tended to evaluate also American scenes as less complex than American participants, likely due to their habit to experience complex context; in a more objective analysis on pictures of Asian and American cities, the first contained a higher number of bounded objects, and big cities had a larger number of bounded objects than middle and small-sized cities. In a subsequent, more implicit task, participants were submitted to a change blindness task, i.e. they had to detect differences between two scenes presented in sequence; the two scenes differed either in focal objects (e.g. truck) or background objects (e.g. 
building, sky). Results revealed that, when Japanese scenes were presented as primes, both Japanese and American participants attended more to the context. The results of this study are informative since they show that Asian participants are more used to complex situations, and that cultural differences in the physical environment influence perception of affordances.

\subsection{Summary}

In this brief session I have focused on the relationship between affordances and culture, highlighting that it would be important to study affordances taking into account the cultural milieu in which they are immersed.

\section{Conclusion}

Affordances are what the environment provides or offers to organisms. However, our environment is full of affordances, thus of action possibilities. In the course of this article we have seen that, when observing objects, multiple action possibilities are activated, for a variety of reasons (see Chinellato \& del Pobil, 2016, for a study highlighting the flexibility of affordances from the point of view of neurorobotics). The same object can evoke different affordances depending on the planned action (e.g. the beak of a teapot can be used to lift it, the handle can activate a more stable grip useful to pour tea), on the distance of the object from us (affordances emerge when objects are near, not when they are out of reach, e.g. Costantini et al., 2011) the situation (e.g. a glass can activate a manipulative grip when lunch is finished and it has to be put in the sink, and a functional grip when used to drink, e.g. lacoboni et al., 2005), on the physical context (e.g. a cork can activate a precision grip when on a bottle, a power grip when seen in a drawer, e.g. Kalenine et al., 2014), on the presence of further objects (e.g. a spoon can activate a different grip when seen near to a bowl, or to a pairs of scissors; e.g., Borghi et al., 2012), on its active vs. passive role in a pair (e.g. a spoon can activate different affordances to right-handed participants when put on the right side of a bowl, compared to when it is on the left side of the bowl, e.g. Yoon et al., 2010), on biomechanical constraints (Jacquet et al., 2013). In all these cases the brain processes sensory information activating a competition between possible actions (Cisek, 2007). This competition is solved dynamically: it will be won by the affordances more relevant to the planned action, that are closer to us, that evoke biomechanically simple actions, that are relevant to the situation at hand and to the physical context: if we consider grasping actions, move contexts will activate more power grips, and use context precision grips. When two objects are presented, the competition will be won by the affordances of the active object in a pair (Xu et al., 2015).

The situation becomes more complex when not only one agent is present on the scene, but multiple agents. Others might be our friends or unknown people (Gianelli et al., 2013a), they might be competitive or collaborative (Becchio et al., 2008b), they can be gentle or rude with us (Di Cesare et al., 2017). Others might simply be present near the object (e.g. Tversky \& Hard, 2012); alternatively, they might be going to interact with the object themselves (Bloesch et al., 2012; Ellis et al., 2013) or say they are going to grasp it (Gianelli et al., 2013a). In these cases a competition between the affordances evoked by the object for us and the affordances evoked for 
the others might take place. Again, it will be solved online, dynamically: the other can be perceived as a competitor, leading us to be faster in reaching and grasping the object, or as a collaborator, leading us to be accurate and careful in dealing with the object. This accuracy characterizes exchanges in which others who are friends, collaborative and gentle with us and others ask for the object (e.g. Sartori et al., 2009), or give it/ receive it (Ferri et al., 2011; Becchio et al., 2008; Scorolli et al., 2014). When we perform a joint action with someone on an object, then we are more prone to provide signals to the other when the relationship is positive and smooth (Sacheli et al., 2011). Finally, the situation might be even more complex, because the object can be valuable and thus elicits a competition, or because we know, or infer, that the object belongs to someone else, and this might block the activation of its affordances (Constable et al., 2011; Scorolli et al., 2017).

In sum: In this paper I intended to make a very simple point: our world is crowded - it is populated by objects, entities, people. Hence, affordance activation is highly flexible and can be influenced by the physical context, by the social context, by the linguistic context, by social norms, and by culture. Affordances should be studied taking in consideration all these aspects. I have reviewed recent, novel evidence highlighting this important fact.

I propose that in all cases, a competition is activated, between the affordances the object would evoke per se and the affordances relevant for the current situation and context. The degree of uncertainty deriving from this competition varies depending on the complexity of the situation. Affordances might be adjusted online, varying depending on the relationship with the others. We might block affordances activation either because the objects are dangerous, or broken, or because we are sensitive to their ownership: we do not respond if the object belongs to someone else. There might be cases in which sensitivity to affordances varies depending on the culture in which we grew up. Further research is needed, to investigate how the presence of multiple affordances of the same object, the presence of multiple objects, and the presence of multiple social agents and of different social norms influences responses. Understanding in depth the complexity of these dynamics and highlighting how exquisitely flexible affordances are represent in my opinion the greatest challenge for future research in this field. 


\section{References}

Ambrosini, E., Scorolli, C., Borghi, A. M., \& Costantini, M. (2012). Which body for embodied cognition? Affordance and language within actual and perceived reaching space. Consciousness and cognition, 21(3), 1551-1557.

Anelli, F., Borghi, A. M., \& Nicoletti, R. (2012). Grasping the pain: motor resonance with dangerous affordances. Consciousness and cognition, 21(4), 1627-1639.

Anelli, F., Nicoletti, R., Bolzani, R., \& Borghi, A. M. (2013a). Keep away from danger: dangerous objects in dynamic and static situations. Frontiers in human neuroscience, 7.

Anelli, F., Ranzini, M., Nicoletti, R., \& Borghi, A. M. (2013b). Perceiving object dangerousness: an escape from pain?. Experimental brain research, 228(4), 457-466.

Barsalou, L. W. (2008). Grounded cognition. Annu. Rev. Psychol., 59, 617-645.

Becchio, C., Sartori, L., Bulgheroni, M., \& Castiello, U. (2008a). The case of Dr. Jekyll and Mr. Hyde: a kinematic study on social intention. Consciousness and cognition, 17(3), 557-564.

Becchio, C., Sartori, L., Bulgheroni, M., \& Castiello, U. (2008b). Both your intention and mine are reflected in the kinematics of my reach-to-grasp movement. Cognition, 106(2), 894-912.

Becchio, C., Sartori, L., \& Castiello, U. (2010). Toward you: The social side of actions. Current Directions in Psychological Science, 19(3), 183-188.

Binkofski, F., \& Buxbaum, L. J. (2013). Two action systems in the human brain. Brain and language, $127(2), 222-229$.

Bjørgen, K. (2016). Physical activity in light of affordances in outdoor environments: qualitative observation studies of 3-5 years olds in kindergarten. Springerplus 5, 950. doi: 10.1186/s40064016-2565-y

Bloesch, E. K., Davoli, C. C., Roth, N., Brockmole, J. R., \& Abrams, R. A. (2012). Watch this! Observed tool use affects perceived distance. Psychonomic Bulletin \& Review, 19(2), 177-183.

Borghi, A. M., Bonfiglioli, C., Lugli, L., Ricciardelli, P., Rubichi, S., \& Nicoletti, R. (2007). Are visual stimuli sufficient to evoke motor information?: Studies with hand primes. Neuroscience letters, 411(1), 17-21.

Borghi, A.M., Caruana, F. (2015). Embodiment Theory. In: James D. Wright (editor-in-chief), International Encyclopedia of the Social \& Behavioral Sciences,2nd edition, Vol 7. Oxford: Elsevier. pp. 420-426. ISBN: 9780080970868. 
Borghi, A. M., Flumini, A., Natraj, N., \& Wheaton, L. A. (2012). One hand, two objects: emergence of affordance in contexts. Brain and cognition, 80(1), 64-73.

Borghi, A. M., \& Riggio, L. (2009). Sentence comprehension and simulation of object temporary, canonical and stable affordances. Brain Research, 1253, 117-128.

Borghi, A. M., \& Riggio, L. (2015). Stable and variable affordances are both automatic and flexible. Frontiers in human neuroscience, 9.

Brand, R.J., Baldwin, D.A., \& Ashburn, L.A. (2002) Evidence for motionese: modifications in mothers' infant-directed action. Developmental Science, 5, 72-83.

Brand, R. J., \& Shallcross, W. L. (2008). Infants prefer motionese to adult-directed action. Developmental science, 11(6), 853-861.

Bruineberg, J., Chemero, A., \& Rietveld, E. (2018). General ecological information supports engagement with affordances for 'higher'cognition. Synthese, 1-21.

Bub, D. N., Masson, M. E. J., \& Cree, G. S. (2008). Evocation of functional and volumetric gestural knowledge by objects and words. Cognition, 106, 27-58.

Buccino, G., Sato, M., Cattaneo, L., Rodà, F., \& Riggio, L. (2009). Broken affordances, broken objects: a TMS study. Neuropsychologia, 47(14), 3074-3078.

Buxbaum, L. J. (2017). Learning, remembering, and predicting how to use tools: Distributed neurocognitive mechanisms: Comment on Osiurak and Badets (2016).

Buxbaum, L. J., \& Kalénine, S. (2010). Action knowledge, visuomotor activation, and embodiment in the two action systems. Annals of the New York Academy of Sciences, 1191(1), 201-218.

Candidi, M., Sacheli, L. M., Era, V., Canzano, L., Tieri, G., \& Aglioti, S. M. (2017). Come together: human-avatar on-line interactions boost joint-action performance in apraxic patients. Social cognitive and affective neuroscience, 12(11), 1793-1802.

Candidi, M., Curioni, A., Donnarumma, F., Sacheli, L. M., \& Pezzulo, G. (2015). Interactional leaderfollower sensorimotor communication strategies during repetitive joint actions. Journal of the Royal Society Interface, 12(110), 20150644.

Cardellicchio, P., Sinigaglia, C., \& Costantini, M. (2012). Grasping affordances with the other's hand: a TMS study. Social cognitive and affective neuroscience, 8(4), 455-459.

Casasanto, D. (2008). Who's afraid of the big bad Whorf? Crosslinguistic differences in temporal language and thought. Language learning, 58(s1), 63-79.

Chemero, A. (2001). What We Perceive When We Perceive Affordances: Commentary on Michaels (2000)" Information, Perception, and Action". Ecological Psychology, 13(2), 111-116. 
Chemero, A. (2003). An outline of a theory of affordances. Ecological psychology, 15(2), 181-195.

Chemero, A. (2009). Radical Embodied Cognitive Science. A Bradford Book.

Chen, M., \& Bargh, J. A. (1999). Consequences of automatic evaluation: Immediate behavioral predispositions to approach or avoid the stimulus. Personality and social psychology bulletin, 25(2), 215-224.

Chinellato, E., \& del Pobil, A. P. (2016). The visual neuroscience of robotic grasping. Achieving sensorimotor skills through dorsal-ventral stream integration. Berlin: Springer International Publishing.

Cisek, P. (2007). Cortical mechanisms of action selection: the affordance competition hypothesis. Philosophical Transactions of the Royal Society of London B: Biological Sciences, 362(1485), 15851599.

Clark, C., \& Uzzell, D. L. (2006). 11 The socio-environmental affordances of adolescents' environments. Children and their environments: Learning, using and designing spaces, 176.

Coello, Y., Bourgeois, J., \& lachini, T. (2012). Embodied perception of reachable space: how do we manage threatening objects?. Cognitive processing, 13(1), 131-135.

Constable, M. D., Kritikos, A., \& Bayliss, A. P. (2011). Grasping the concept of personal property. Cognition, 119(3), 430-437.

Constable, M. D., Kritikos, A., Lipp, O. V., \& Bayliss, A. P. (2014). Object ownership and action: The influence of social context and choice on the physical manipulation of personal property.

Experimental brain research, 232(12), 3749-3761.

Constable, M. D., Bayliss, A. P., Tipper, S. P., Spaniol, A. P., Pratt, J., \& Welsh, T. N. (2016).

Ownership status influences the degree of joint facilitatory behavior. Psychological science, 27(10), 1371-1378.

Costantini, M., Ambrosini, E., Tieri, G., Sinigaglia, C., \& Committeri, G. (2010). Where does an object trigger an action? An investigation about affordances in space. Experimental brain research, 207(1-2), 95-103.

Costantini, M., Ambrosini, E., Scorolli, C., \& Borghi, A. M. (2011a). When objects are close to me: affordances in the peripersonal space. Psychonomic bulletin \& review, 18(2), 302-308.

Costantini, M., Committeri, G., \& Sinigaglia, C. (2011b). Ready both to your and to my hands: Mirroring the reaching space of others. PLOS ONE, 6.

Costantini, M., \& Sinigaglia, C. (2011). 17 Grasping Affordance: A Window onto Social Cognition. Joint attention: New developments in psychology, philosophy of mind, and social neuroscience, 431. 
Creem-Regehr, S. H., Gagnon, K. T., Geuss, M. N., \& Stefanucci, J. K. (2013). Relating spatial perspective taking to the perception of other's affordances: providing a foundation for predicting the future behavior of others. Frontiers in human neuroscience, 7.Ellis et al., 2013

de Haan, E. H. F., Jackson, S. T., \& Schenk, T. (2018). Where are we now with 'What' and 'How'? Cortex, 98(1), 7. https://doi.org/10.1016/j.rehab.2017.02.002.

De Stefani, E., De Marco, D., \& Gentilucci, M. (2016). The Effects of Meaning and Emotional Content of a Sentence on the Kinematics of a Successive Motor Sequence Mimiking the Feeding of a Conspecific. Frontiers in psychology, 7.

Di Cesare, G., De Stefani, E., Gentilucci, M., \& De Marco, D. (2017). Vitality forms expressed by others modulate our own motor response: a kinematic study. Frontiers in human neuroscience, 11, 565.

Ellis, R., Swabey, D., Bridgeman, J., May, B., Tucker, M., \& Hyne, A. (2013). Bodies and other visual objects: the dialectics of reaching toward objects. Psychological research, 77(1), 31-39.

Ferretti, G. (2016a). Pictures, action properties and motor related effects. Synthese, 193(12), 37873817.

Ferretti, G. (2016b). Visual Feeling of Presence. Pacific Philosophical Quarterly. DOI: 10.1111/papq.12170.

Ferretti, G. (2016c). Through the Forest of Motor Representations. Consciousness and Cognition, 43, pp. 177-196. DOI: http://dx.doi.org/10.1016/j.concog.2016.05.013.

Ferretti, G. (2017a). Are Pictures Peculiar Objects of Perception? Journal of the American Philosophical Association, 3(3), pp. 372-393. DOI: 10.1017/apa.2017.28.

Ferretti, G. (2017b). Two Visual Systems in Molyneux Subjects. Phenomenology and the Cognitive Sciences, 17(4), pp. 643-679. DOI: https://doi.org/10.1007/s11097-017-9533-z.

Ferretti, G. (2018). The Neural Dynamics of Seeing-In. Erkenntnis, DOI: https://doi.org/10.1007/s10670-018-0060-2.

Ferri, F., Campione, G. C., Dalla Volta, R., Gianelli, C., \& Gentilucci, M. (2010). To me or to you? When the self is advantaged. Experimental brain research, 203(4), 637-646.

Ferri, F., Campione, G. C., Dalla Volta, R., Gianelli, C., \& Gentilucci, M. (2011). Social requests and social affordances: how they affect the kinematics of motor sequences during interactions between conspecifics. PLoS One, 6(1), e15855.

Fini, C., Bardi, L., Epifanio, A., Committeri, G., Moors, A., \& Brass, M. (2017). Transcranial direct current stimulation (tDCS) of the inferior frontal cortex affects the "social scaling" of extrapersonal space depending on perspective-taking ability. Experimental brain research, 235(3), 673-679. 
Fini, C., Costantini, M., \& Committeri, G. (2014). Sharing space: the presence of other bodies extends the space judged as near. PloS one, 9(12), e114719.

Fini, C., Committeri, G., Müller, B. C., Deschrijver, E., \& Brass, M. (2015). How watching Pinocchio movies changes our subjective experience of extrapersonal space. PloS one, 10(3), e0120306.

Freud, E., Macdonald, S. N., Chen, J., Quinlan, D. J., Goodale, M. A., \& Culham, J. C. (2018). Getting a grip on reality: Grasping movements directed to real objects and images rely on dissociable neural representations. Cortex, 98, 34-48.

Gallese, V., \& Lakoff, G. (2005). The brain's concepts: The role of the sensory-motor system in conceptual knowledge. Cognitive neuropsychology, 22(3-4), 455-479.

Gianelli, C., Lugli, L., Baroni, G., Nicoletti, R., \& Borghi, A. M. (2011). "The object is wonderful or prickly": how different object properties modulate behavior in a joint context,". European Perspectives on Cognitive Science, 978-954.

Gianelli, C., Scorolli, C., \& Borghi, A. M. (2013a). Acting in perspective: the role of body and language as social tools. Psychological Research, 77(1), 40-52.

Gianelli, C., Lugli, L., Baroni, G., Nicoletti, R., \& Borghi, A. M. (2013b). The impact of social context and language comprehension on behaviour: a kinematic investigation. PloS one, 8(12), e85151.

Gibson, J. J. (1979). The Ecological Approach to Visual Perception. Boston: Houghton Mifflin.

Glenberg, A. (2014). Few believe the world is flat. Canadian Journal of Experimental Psychology, 68(4), 250.

Golonka, S. (2015). Laws and Conventions in Language-Related Behaviors. Ecological Psychology, 27(3), 236-250.

Grèzes, J., Armony, J. L., Rowe, J., \& Passingham, R. E. (2003). Activations related to "mirror" and "canonical" neurones in the human brain: an fMRI study. Neuroimage, 18(4), 928-937.

Heft, H. (1989). Affordances and the body: An intentional analysis of Gibson's ecological approach to visual perception. Journal for the Theory of Social Behavior, 19, 1-30

Heft, H. (2003). Affordances, Dynamic Experience, and the Challenge of Reification. Ecological Psychology, 15(2), 149-180.

Iacoboni, M., Molnar-Szakacs, I., Gallese, V., Buccino, G., Mazziotta, J. C., \& Rizzolatti, G. (2005). Grasping the intentions of others with one's own mirror neuron system. PLoS biology, 3(3), e79.

Jacquet, P. O., Chambon, V., Borghi, A. M., \& Tessari, A. (2012). Object affordances tune observers' prior expectations about tool-use behaviors. PloS one, 7(6), e39629.

Jax, S. A., \& Buxbaum, L. J. (2010). Response interference between functional and structural actions linked to the same familiar object. Cognition, 115(2), 350-355. 
Jongeneel, D., Withagen, R., \& Zaal, F. T. (2015). Do children create standardized playgrounds? A study on the gap-crossing affordances of jumping stones.Journal of Environmental Psychology, 44, 45-52.

Kalénine, S., Shapiro, A. D., Flumini, A., Borghi, A. M., \& Buxbaum, L. J. (2014). Visual context modulates potentiation of grasp types during semantic object categorization. Psychonomic bulletin \& review, 21(3), 645-651.

Kalénine, S., \& Buxbaum, L. J. (2016). Thematic knowledge, artifact concepts, and the left posterior temporal lobe: Where action and object semantics converge. Cortex,82, 164-178.

Kalénine, S., Wamain, Y., Decroix, J., \& Coello, Y. (2016). Conflict between object structural and functional affordances in peripersonal space. Cognition, 155, 1-7.

Liu, P., Cao, R., Chen, X., \& Wang, Y. (2017). Response inhibition or evaluation of danger? An event-related potential study regarding the origin of the motor interference effect from dangerous objects. Brain Research, 1664, 63-73.

Liu, P. (2018). Time-frequency analysis of event-related potentials associated with the origin of the motor interference effect from dangerous objects. Brain research.

Michaels, C. F. (2000). Information, perception, and action: What should ecological psychologists learn from Milner and Goodale (1995)?. Ecological Psychology, 12(3), 241-258.

Miyamoto, Y., Nisbett, R. E., \& Masuda, T. (2006). Culture and the physical environment: Holistic versus analytic perceptual affordances. Psychological Science, 17(2), 113-119.

Murata, A., Gallese, V., Luppino, G., Kaseda, M., \& Sakata, H. (2000). Selectivity for the shape, size, and orientation of objects for grasping in neurons of monkey parietal area AIP. Journal of neurophysiology, 83(5), 2580-2601.

Natraj, N., Poole, V., Mizelle, J. C., Flumini, A., Borghi, A. M., \& Wheaton, L. A. (2013). Context and hand posture modulate the neural dynamics of tool-object perception. Neuropsychologia, 51(3), 506-519.

Natraj, N., Pella, Y. M., Borghi, A. M., \& Wheaton, L. A. (2015). The visual encoding of tool-object affordances. Neuroscience, 310, 512-527.

Neldner, K., Mushin, I., \& Nielsen, M. (2017). Young children's tool innovation across culture: Affordance visibility matters. Cognition, 168, 335-343.

Newman-Norlund, R. D., van Schie, H. T., van Zuijlen, A. M. J., \& Bekkering, H. (2007). The mirror neuron system is more active during complementary compared with imitative action. Nature Neuroscience, $10,817-818$. 
Norman, D. A. (1999). Affordance, conventions, and design. interactions, 6(3), 38-43.

Orban, G. A., \& Caruana, F. (2014). The neural basis of human tool use. Frontiers in psychology, 5, 310.

Osiurak, F., \& Badets, A. (2016). Tool use and affordance: Manipulation-based versus reasoningbased approaches. Psychological review, 123(5), 534.

Pellicano, A., lani, C., Borghi, A. M., Rubichi, S., \& Nicoletti, R. (2010). Simon-like and functional affordance effects with tools: The effects of object perceptual discrimination and object action state. The Quarterly Journal of Experimental Psychology, 63(11), 2190-2201.

Pezzulo, G., lodice, P., Donnarumma, F., Dindo, H., \& Knoblich, G. (2017). Avoiding accidents at the champagne reception: A study of joint lifting and balancing. Psychological science, 28(3), 338-345.

Pezzulo, G., \& Cisek, P. (2016). Navigating the affordance landscape: feedback control as a process model of behavior and cognition. Trends in cognitive sciences, 20(6), 414-424.

Raymond CM, Kyttä M and Stedman R (2017) Sense of Place, Fast and Slow: The Potential Contributions of Affordance Theory to Sense of Place. Front. Psychol. 8:1674. doi: 10.3389/fpsyg.2017.01674

Reed, E. S. (1996). Encountering the world. New York: Oxford University Press.

Riddoch, M. J., Pippard, B., Booth, L., Rickell, J., Summers, J., Brownson, A., \& Humphreys, G. W. (2011). Effects of action relations on the configural coding between objects. Journal of Experimental Psychology: Human Perception and Performance, 37(2), 580.

Rietveld, E., \& Kiverstein, J. (2014). A rich landscape of affordances. Ecological Psychology, 26(4), 325-352.

Rizzolatti, G., \& Matelli, M. (2003). Two different streams form the dorsal visual system: anatomy and functions. Experimental brain research, 153(2), 146-157.

Roberts, K. L., \& Humphreys, G. W. (2011). Action relations facilitate the identification of brieflypresented objects. Attention, Perception, \& Psychophysics, 73(2), 597-612.

Sacheli, L. M., Candidi, M., Pavone, E. F., Tidoni, E., \& Aglioti, S. M. (2012). And yet they act together: interpersonal perception modulates visuo-motor interference and mutual adjustments during a joint-grasping task. PloS one, 7(11), e50223.

Sacheli, L. M., Tidoni, E., Pavone, E. F., Aglioti, S. M., \& Candidi, M. (2013). Kinematics fingerprints of leader and follower role-taking during cooperative joint actions. Experimental brain research, 226(4), 473-486.

Sakata, H., Taira, M., Murata, A., \& Mine, S. (1995). Neural mechanisms of visual guidance of hand action in the parietal cortex of the monkey. Cerebral Cortex, 5(5), 429-438. 
Sakreida, K., Effnert, I., Thill, S., Menz, M. M., Jirak, D., Eickhoff, C. R., ... \& Binkofski, F. (2016). Affordance processing in segregated parieto-frontal dorsal stream sub-pathways. Neuroscience \& Biobehavioral Reviews, 69, 89-112.

Sartori, L., Becchio, C., Bulgheroni, M., \& Castiello, U. (2009). Modulation of the action control system by social intention: unexpected social requests override preplanned action. Journal of Experimental Psychology: Human Perception and Performance, 35(5), 1490.

Scorolli, C., Miatton, M., Wheaton, L. A., \& Borghi, A. M. (2014). I give you a cup, I get a cup: a kinematic study on social intention. Neuropsychologia, 57, 196-204.

Scorolli, C., Borghi, A. M., \& Tummolini, L. (2017). Cues of control modulate the ascription of object ownership. Psychological Research, 1-26.

Sevos J, Grosselin A, Brouillet D, Pellet J and Massoubre C (2016) Is there any Influence of Variations in Context on Object-Affordance Effects in Schizophrenia? Perception of Property and Goals of Action. Front. Psychol. 7:1551. doi: 10.3389/fpsyg.2016.01551

Stoffregen, T. A. (2003). Affordances as properties of the animal-environment system. Ecological psychology, 15(2), 115-134.

Thill, S., Caligiore, D., Borghi, A. M., Ziemke, T., \& Baldassarre, G. (2013). Theories and computational models of affordance and mirror systems: an integrative review. Neuroscience \& Biobehavioral Reviews, 37(3), 491-521.

Tipper, S. P., Paul, M. A., \& Hayes, A. E. (2006). Vision-for-action: The effects of object property discrimination and action state on affordance compatibility effects. Psychonomic bulletin \& review, 13(3), 493-498.

Tucker, M., \& Ellis, R. (1998). On the relations between seen objects and components of potential actions. Journal of Experimental Psychology: Human perception and performance, 24(3), 830.

Tucker, M., \& Ellis, R. (2001). The potentiation of grasp types during visual object categorization. Visual cognition, 8(6), 769-800.

Tucker, M., \& Ellis, R. (2004). Action priming by briefly presented objects. Acta psychologica, $116(2), 185-203$.

Tummolini, L., Scorolli, C., \& Borghi, A. M. (2013). Disentangling the sense of ownership from the sense of fairness. Behavioral and Brain Sciences, 36(1), 101-102.

Turvey,M. (1992). Affordances and prospective control: An outline of the ontology. Ecological Psychology, $4,173-187$ 
Tversky, B., \& Hard, B.M. (2009). Embodied and disembodied cognition: Spatial perspectivetaking. Cognition, 110(1), 124-129.

van Elk, M., van Schie, H., \& Bekkering, H. (2014). Action semantics: a unifying conceptual framework for the selective use of multimodal and modality-specific object knowledge. Physics of life reviews, 11(2), 220-250.

Yoon, E. Y., Humphreys, G. W., \& Riddoch, M. J. (2010). The paired-object affordance effect. Journal of Experimental Psychology: Human Perception and Performance, 36(4), 812.

Young, G. (2006). Are different affordances subserved by different neural pathways?. Brain and cognition, 62(2), 134-142.

Witt, J. K., \& Riley, M. (2014). Discovering your inner Gibson: Reconciling action-specific and ecological approaches Journal of Experimental Psychology: Human Perception and Performance, 1353-1370.

Withagen, R., \& Caljouw, S. R. (2017). Aldo van Eyck's Playgrounds: Aesthetics, Affordances, and Creativity. Frontiers in psychology, 8, 1130.

Wokke, M. E., Knot, S. L., Fouad, A., \& Ridderinkhof, K. R. (2016). Conflict in the kitchen: Contextual modulation of responsiveness to affordances. Consciousness and cognition, 40, 141146.

Xu, S., Humphreys, G. W., \& Heinke, D. (2015). Implied actions between paired objects lead to affordance selection by inhibition. Journal of Experimental Psychology: Human Perception and Performance, 41(4), 1021.

Xu, S., Humphreys, G. W., Mevorach, C., \& Heinke, D. (2017). The involvement of the dorsal stream in processing implied actions between paired objects: A TMS study. Neuropsychologia, 95, 240249.

Zipoli Caiani, S. (2014). Extending the notion of affordance. Phenomenology and the Cognitive Sciences, 13(2), 275-293.

Zipoli Caiani, S., \& Ferretti, G. (2017). Semantic and pragmatic integration in vision for action. Consciousness and cognition, 48, 40-54. 